\title{
APRIL secreted by neutrophils binds to heparan sulfate proteoglycans to create plasma cell niches in human mucosa
}

\author{
Bertrand Huard,1,2 Thomas McKee, ${ }^{3}$ Carine Bosshard, ${ }^{1,2}$ Stéphane Durual, ${ }^{1}$ Thomas Matthes, ${ }^{1}$ \\ Samir Myit, ${ }^{3}$ Olivier Donze, ${ }^{4}$ Christophe Frossard, ${ }^{5}$ Carlo Chizzolini, ${ }^{5}$ Christiane Favre, ${ }^{1}$ \\ Rudolf Zubler, ${ }^{1}$ Jean Philippe Guyot, ${ }^{6}$ Pascal Schneider, ${ }^{7}$ and Eddy Roosnek ${ }^{1}$ \\ 'Division of Hematology, Geneva University Hospital, Geneva, Switzerland. 2Department of Patho-Immunology and \\ ${ }^{3}$ Department of Pathology, Geneva University Medical Center, Geneva, Switzerland. ${ }^{4}$ Apotech Corporation, Epalinges, Switzerland. \\ ${ }^{5}$ Division of Immunology and Allergology and ${ }^{6}$ Department of Otorhinolaryngology, Geneva University Hospital, Geneva, Switzerland. \\ ${ }^{7}$ Biochemistry Department, University of Lausanne, Epalinges, Switzerland.
}

\begin{abstract}
The bone marrow constitutes a favorable environment for long-lived antibody-secreting plasma cells, providing blood-circulating antibody. Plasma cells are also present in mucosa-associated lymphoid tissue (MALT) to mediate local frontline immunity, but how plasma cell survival there is regulated is not known. Here we report that a proliferation-inducing ligand (APRIL) promoted survival of human upper and lower MALT plasma cells by upregulating expression of the antiapoptotic proteins bcl-2, bcl- $\mathrm{x}_{\mathrm{L}}$, and $\mathrm{mcl}-1$. The in situ localization of APRIL was consistent with such a prosurvival role in MALT. In upper MALT, tonsillar epithelium produced APRIL. Upon infection, APRIL production increased considerably when APRIL-secreting neutrophils recruited from the blood infiltrated the crypt epithelium. Heparan sulfate proteoglycans (HSPGs) retained secreted APRIL in the subepithelium of the infected zone to create APRIL-rich niches, wherein IgG-producing plasma cells accumulated. In lower MALT, neutrophils were the unique source of APRIL, giving rise to similar niches for IgA-producing plasmocytes in villi of lamina propria. Furthermore, we found that mucosal humoral immunity in APRIL-deficient mice is less persistent than in WT mice. Hence, production of APRIL by inflammation-recruited neutrophils may create plasma cell niches in MALT to sustain a local antibody production.
\end{abstract}

\section{Introduction}

$\mathrm{B}$ cells encountering antigen in the $\mathrm{T}$ cell zone of secondary lymphoid organs migrate into primary follicles to divide extensively. After this initial proliferation, B cells have to process and present antigen to $\mathrm{T}$ cells to receive signals for Ig class switch recombination and somatic hypermutations, both mechanisms being under the tight control of a deaminase acting on cytidine present in Ig genes (1). The latter alters their affinity for the antigen. B cells harboring Igs with a too low affinity will not be able to compete for survival signals presented as trapped antigens in the light zone of germinal centers and undergo apoptosis. This selection process, facilitated by the open structure of germinal centers (2), ensures that only B cells expressing the highest affinity antibodies will receive sufficient survival signals and leave the germinal center as plasmablasts. After leaving the induction zone, plasmablasts must find an appropriate environment to terminate their differentiation and survive as plasma cells. The bone marrow is a favorable environment for plasma cells (3), because it expresses the cytokine IL-6, the ligand(s) for the adhesion molecule CD44, and the chemokine SDF-1 (4). Alternatively, plasmablasts home to a mucosa-associated lymphoid tissue (MALT), usually the site where they initially

Nonstandard abbreviations used: ACRP, adipocyte complement-related protein; APRIL, a proliferation-inducing ligand; BAFF, B cell activation factor; BAFF-R, BAFF receptor; BCMA, B cell maturation antigen; HSPG, heparan-sulfate proteoglycan; MALT, mucosa-associated lymphoid tissue; TACI, transmembrane activator, calcium modulator, and cyclophilin ligand interactor.

Conflict of interest: Olivier Donze is employed by Apotech Corporation, whose products were used in the present study.

Citation for this article: J. Clin. Invest. 118:2887-2895 (2008). doi:10.1172/JCI33760. encountered the antigen (5). This occurs in particular when the MALT is inflamed (6). In contrast to the bone marrow, the factors that sustain plasma cell survival in MALT are not known.

A proliferation-inducing ligand (APRIL, also known as TNFSF13) and the $\mathrm{B}$ cell activation factor (BAFF, also known as TNFSF13B) are 2 closely related members of the TNF superfamily that share the transmembrane activator, calcium modulator, and cyclophilin ligand interactor (TACI, also known as TNFRSF13B) and the B cell maturation antigen (BCMA, also known as TNFRSF17) as canonical TNF-like receptors (7). Two recent reports suggested that these molecules contribute to survival of bone-marrow plasma cells. Blocking of APRIL/BAFF (8) as well as genetic deletion of BCMA (9) impairs the number of plasma cells recovered in the bone marrow after immunization. Although APRIL and BAFF share many characteristics, the differences with respect to receptor binding are considerable. BCMA binds APRIL with a 2-logs higher affinity than BAFF (10). BAFF but not APRIL binds to BAFF receptor (BAFF-R, also known as TNFRSF13C) (11), while only APRIL binds to heparan-sulfate proteoglycans (HSPGs) $(8,12)$. These differences are likely to explain the nonredundant functions of these 2 TNF ligands. BAFF (13) but not APRIL (14) is prerequisite for the generation and maintenance of a mature $B$ cell compartment. The role of APRIL is more restricted to late stage of B cell differentiation, since APRIL-deficient mice have only impaired IgA and IgA/IgM antibody responses to T cell-dependent and -independent mucosal antigens, respectively (15).

In mucosa, the epithelium plays an important role in local antibody responses. Indeed, mucosal epithelial cells stimulated by pathogens via their TLRs produce plasma cell chemokines (5). This chemoattraction explains the subepithelial homing for mucosal plasma cells. 
A
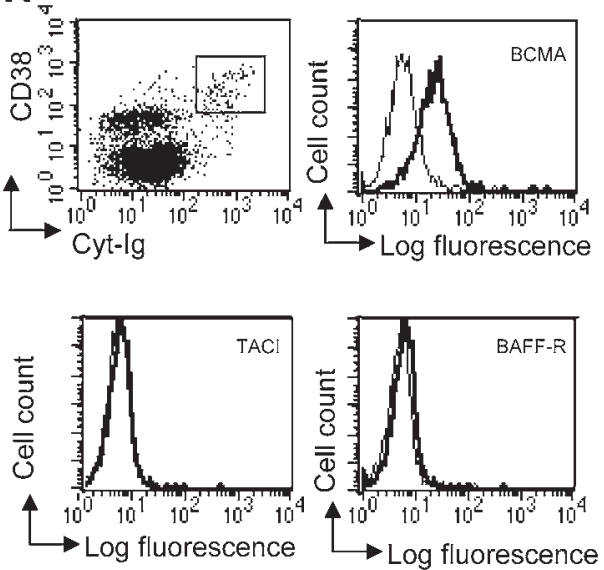

c

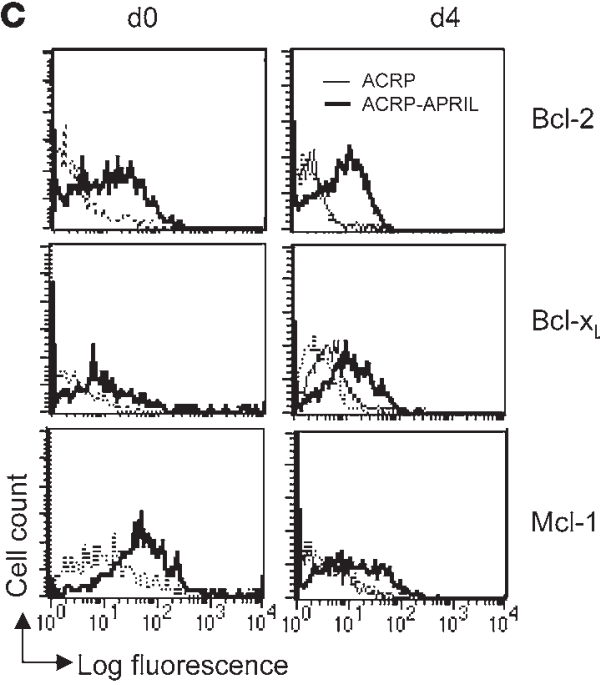

B
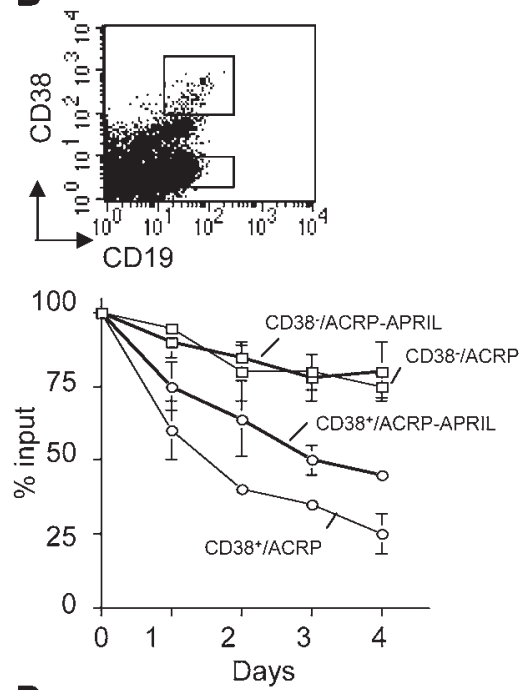

D

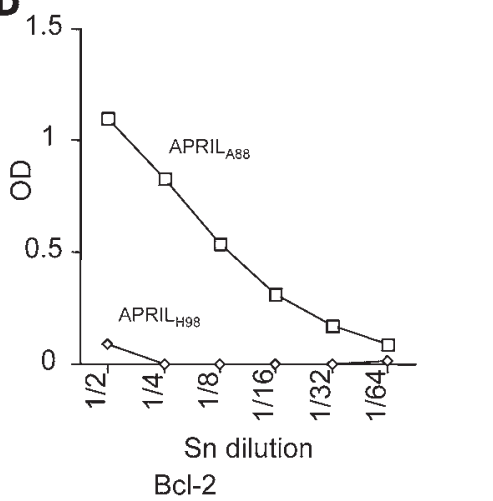

Figure 1

In vitro prosurvival role for APRIL in tonsillar plasma cells. (A) APRIL-receptor expression on tonsillar plasma cells. The histograms show the expression of the indicated receptors on cells from the $C D 19+C D 38^{\text {high }}$ cytosplasmic-lg $\mathrm{g}^{+}\left(\mathrm{Cyt}-\mathrm{Ig}^{+}\right)$subset in the top left dot plot (rectangle). Similar results were obtained with tonsil cell suspensions from 8 patients. (B) $\mathrm{CD}_{19}{ }^{+} \mathrm{CD} 38^{+}$and CD19+CD38 low/- tonsillar B cells (top panel, rectangles) were purified and incubated in medium containing ACRP-APRIL or control ACRP, both at $3 \mu \mathrm{g} / \mathrm{ml}$. The number of viable cells was counted by trypan-blue exclusion every day for 4 days (bottom panel). Data are presented as mean $\pm S D$. (C) Intracellular expression of bcl-2, bcl- $\mathrm{x}_{\mathrm{L}}$, and $\mathrm{mcl}-1$ was analyzed by flow cytometry on purified $\mathrm{CD} 19^{+} \mathrm{CD} 38^{+}$at the initiation of the culture and after 4 days of culture with ACRP-APRIL or control ACRP. Dotted lines correspond to isotype control staining. (D) Binding of APRIL ${ }_{\mathrm{A} 88}$ and APRIL $\mathrm{H}_{\mathrm{H} 8}$ to plastic-coated HSPG $(1 \mu \mathrm{g} / \mathrm{ml})$ was revealed with the Aprily-5 mAb $(1 \mu \mathrm{g} / \mathrm{ml})$ (top panel). $\mathrm{CD}_{19}{ }^{+} \mathrm{CD} 38^{+}$tonsillar plasma cells were incubated in plastic wells coated with HSPG- or HSPG-binding APRIL A88 $_{\text {for }}$ 4 days. Intracellular bcl-2 expression was analyzed as in C (bottom panel). Data are representative of 2 independent experiments performed with 2 different donors. Sn, supernatant.

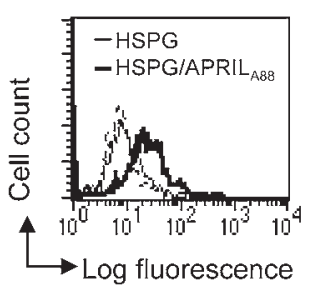

In addition to chemoattraction, the mucosal epithelium can finetune humoral responses by inducing extra-germinal center Ig class switch recombination $(16,17)$. Here we analyzed APRIL expression in human, upper oral and lower gut MALT. We found that MALT-infiltrating neutrophils and the tonsillar epithelium produced APRIL that accumulated on subepithelial HSPG, thereby creating APRILrich niches for plasma cells. The latter is consistent with the role observed in vitro for APRIL in the control of plasma cell survival.

\section{Results}

APRIL provides a survival signal to buman MALT plasma cells. To investigate the role of APRIL in MALT plasma cells, we first analyzed expression of APRIL receptors by tonsillar plasma cells. Since no antibodies were available to date for staining endogenous TACI or BCMA in fixed tissues, we analyzed single cell suspensions isolated from tonsils by flow cytometry. Figure 1A shows that tonsillar plasma cells identified by a CD $19^{+} \mathrm{CD} 38^{\text {high }}$ cytosplasmic-Ig ${ }^{+}$ $\left(\mathrm{Cyt}-\mathrm{Ig}^{+}\right)$phenotype expressed BCMA but were negative for TACI.
This was confirmed with 5 different mAbs against TACI that all stained the TACI ${ }^{+}$IM9 B cell line (18). Of note, BAFF-R was also not expressed, which was consistent with the absence of BAFF-R staining observed in situ on cyt- $\mathrm{Ig}^{+}$tonsillar plasma cells (Supplemental Figure 1; supplemental material available online with this article; doi:10.1172/JCI33760DS1). We next assessed APRIL signaling in tonsillar CD $19^{+} \mathrm{CD} 38^{\text {high }}$ plasma cells. Soluble oligomerized APRIL (adipocyte complement-related protein-APRIL [ACRP-APRIL]) sustained the in vitro survival of purified plasma cells, while no effect was seen on the $\mathrm{CD} 19^{+} \mathrm{CD} 38^{-}$population, consisting mainly of naive B cells (Figure 1B). Tonsillar plasma cells expressed high levels of intracellular bcl-2 (Figure 1C), as previously reported (19), but this expression was readily lost upon in vitro culture unless APRIL was present. Similarly, we observed a sustained expression of the other prosurvival factors bcl- $x_{\mathrm{L}}$ and mcl- 1 in the presence of exogenous APRIL. Because this survival effect was mediated by an engineered form of APRIL that had to be fused to the collagen-based oligomerizing moiety of ACRP30 to be active (8), we wondered 

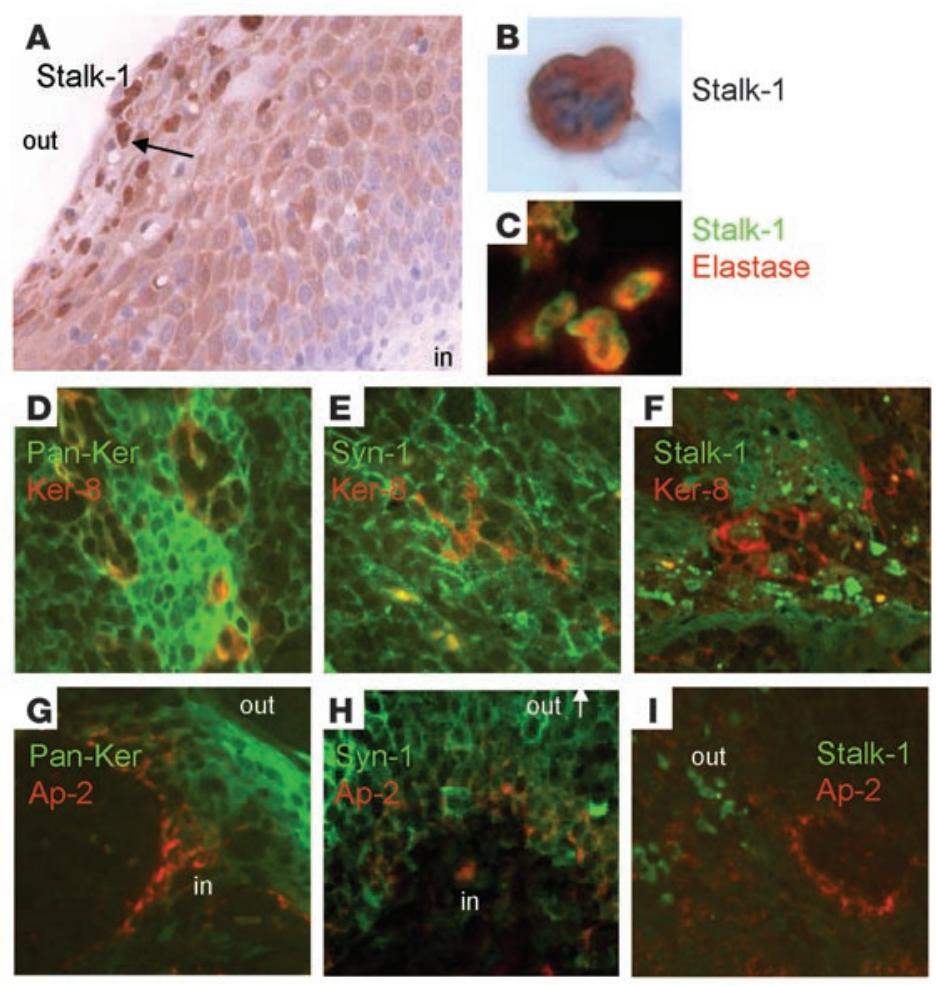

\section{Figure 2}

APRIL-rich niches are formed in subepithelium zone from the tonsillar crypt. (A) Presence of APRIL-producing cells (Stalk-1 staining, $5 \mu \mathrm{g} / \mathrm{ml}$ ) in tonsillar crypt epithelium. The arrow indicates a cell stained brightly. (B) Some Stalk-1-stained cells in the epithelium have a trilobular nucleus. (C) These Stalk-1+ (green) cells coexpress elastase (red, $1 \mu \mathrm{g} / \mathrm{ml}$ ). (D) The crypt epithelium is composed of keratin $8^{+}(1 / 2$, red) cells among other pan-keratin ${ }^{+}(1 / 100$, green) and $(E)$ syndecan-1+ $\left(10 \mu \mathrm{g} / \mathrm{ml}\right.$, green) epithelial cells. (F) Keratin $8^{+}(\mathrm{red})$ and keratin $8^{-}$ crypt epithelial cells produce APRIL (Stalk-1; green). Secreted APRIL (red; Aprily-2 staining, $2 \mu \mathrm{g} / \mathrm{ml}$ ) is retained on (G) pankeratin $^{+}$(green) epithelial cells $(\mathbf{H})$ expressing syndecan-1 (green). The arrow indicates the direction for the crypt lumen. (I) Secreted APRIL (red) accumulates at sites distant from APRIL-producing cells (green) in the subepithelial zone. Original magnification, $\times 20(\mathbf{A}$ and $\mathbf{I}) ; \times 40(\mathbf{C}-\mathbf{H}) ; \times 100(\mathbf{B})$. Pictures shown are representative of more than 10 crypt epithelia from patients with recurrent tonsillitis. in, tonsillar parenchyma; out, crypt lumen; Pan-Ker, pan-keratin+; Ker-8, keratin 8+; Syn-1, syndecan-1+; Ap-2, Aprily-2. whether native trimeric APRIL could promote plasma cell survival after being oligomerized by binding to HSPG. We were not able to test this by binding of APRIL onto HSPG expressed by plasma cells, since we observed that the CD138 core protein carrying plasma cell HSPG is lost during cell isolation as previously reported by others $(20,21)$. Hence, we bound soluble trimeric APRIL onto plastic-coated HSPG. APRIL $\mathrm{A}_{\mathrm{A} 8}$, but not the mutant $\mathrm{APRIL}_{\mathrm{H} 98}$ lacking the HSPG interaction domain, readily bound to coated HSPG, as revealed with the anti-APRIL mAb, Aprily-5 (Figure 1D, top panel). Under these conditions, APRIL $\mathrm{A}_{\mathrm{A} 8}$ sustained intracellular expression of bcl-2 in plasma cells (Figure 1D, bottom panel), showing that APRIL is functional when bound onto HSPG. Taken together, this analysis indicates that APRIL impacts humoral responses at a late stage by providing a survival signal to plasma cells.

APRIL-rich niches are created in MALT. We next studied APRIL expression in MALT with a pair of APRIL-specific antibodies recognizing either APRIL-producing cells (Stalk-1 Ab) or secreted APRIL (Aprily-2 mAb). We already established specificity of this pair of Abs with inhibition of the in situ reactivity by the corresponding antigens and size identification of the detected products by western blot analysis of tissue lysates (22). Figure 2A shows that crypt tonsillar epithelium contained APRIL-producing (Stalk-1 staining) cells. This is specific to tonsil epithelium, since normal human skin does not contain such cells (23). Some cells (Figure 2A, arrow) stained brighter with Stalk-1 than others. High-power microscopy revealed the trilobular nucleus of these cells (Figure 2B), which together with the uniform expression of elastase (Figure 2C) and CD15 (data not shown) identified them as neutrophils. The other Stalk-1-stained cells had a morphology of epithelial cells. Keratin $8^{+}$cells that characterized the tonsillar crypt (24) were mixed with other pan-keratin ${ }^{+}$(Figure 2D) and syndecan $-1^{+}$(Figure 2E) epithelial cells. Keratin $8^{+}$and keratin $8^{-}$crypt epithelial cells produced APRIL (Figure 2F). In these crypts, we observed secreted APRIL (Aprily-2 staining) in the subepithelial zone on pan-keratin ${ }^{+}$epithelial cells (Figure $2 \mathrm{G}$ ), bound to epithelial cells expressing syndecan-1, an APRIL-binding HSPG $(8,12)$ (Figure $2 \mathrm{H}$ ). However, cells that produced APRIL did not stain for secreted APRIL. As a result, this created zones rich in APRIL in the subepithelium, separated from the main production site that located toward the apical lumen (Figure 2I). We observed a similar production and retention of APRIL at the level of the surface epithelium (data not shown). Hence, APRIL secreted by epithelial cells was retained in the subepithelial zone by binding to HSPG, forming APRIL-rich niches.

Plasma cells accumulate in subepithelial APRIL-rich niches of MALT. Plasma cells home close to the epithelium in MALT $(25,26)$. In tonsils, we observed that plasma cells homed to the APRIL-rich niches (Figure 3A) beneath pan-keratin ${ }^{+}$cells (Figure 3B). The yellow color observed at the epithelium level is due to depots of secreted Ig. This homing was specific to plasma cells, since less differentiated CD20 ${ }^{+} \mathrm{B}$ cells (27) were excluded from these zones (Figure $3 C)$. Confocal microscopy demonstrated that secreted APRIL was either retained in the vicinity of or directly on plasma cells (Figure 3D). Accumulating plasma cells expressed syndecan-1 (Figure 3E), allowing them to bind APRIL (Figure 3F, arrow). These plasma cells produced IgG and to a lesser extent IgA, but no IgM (Figure $3 G$ ). We next studied expression of prosurvival factors in these plasma cells. We could not perform 2-color immunofluorescence for $b c l-x_{L}, m c l-1$, and bcl-2 due to their low expression level. However, using an enhanced enzymatic detection assay and staining of serial sections, we observed that subepithelial $\mathrm{IgG}^{+}$plasma cells expressed bcl- $\mathrm{x}_{\mathrm{L}}$, mcl-1, and bcl-2 (Supplemental Figure 2). This in situ analysis confirmed the in vitro sustained expression of these 3 survival factors observed in the presence of exogenous APRIL. It 

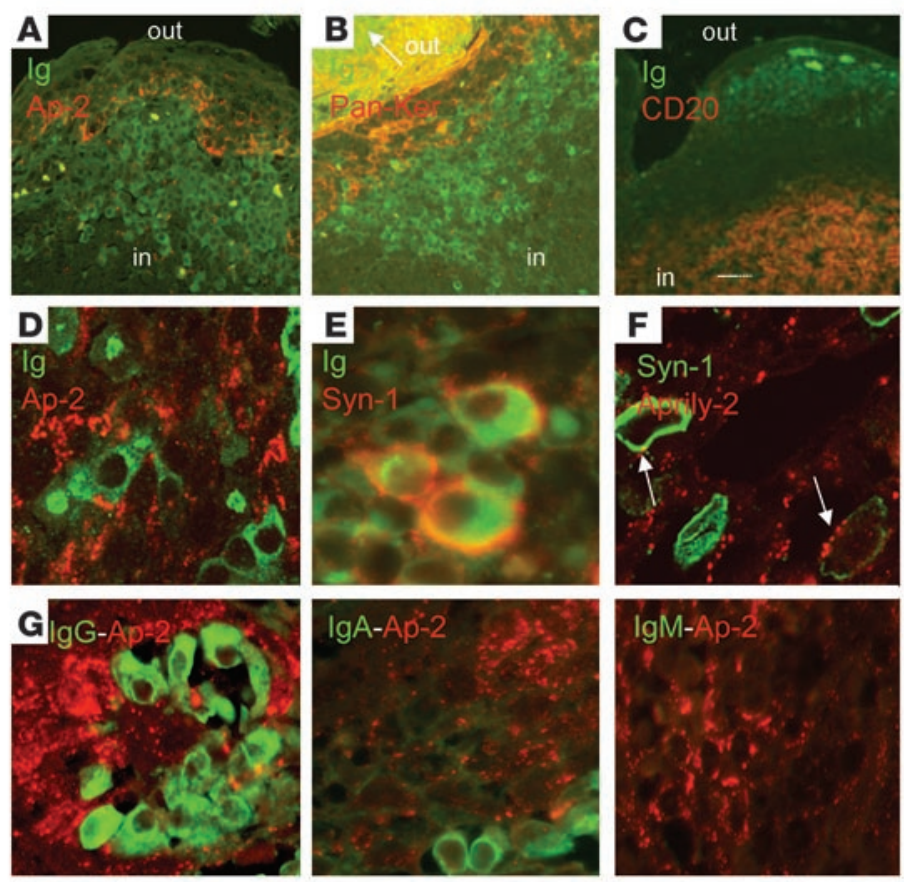

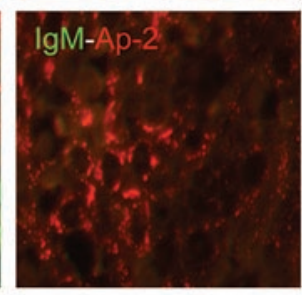

\section{Figure 3}

Plasma cells accumulate in APRIL-rich niches from tonsils. (A and B) Plasma cells (green; Ig staining, $5 \mu \mathrm{g} / \mathrm{ml}$ ) accumulate close to depots of secreted APRIL (red) (A), underneath pan-

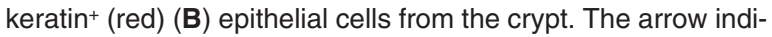
cates the direction for the crypt lumen. (C) CD20+ cells (red; $5 \mu \mathrm{g} / \mathrm{ml}$ ) are excluded from subepithelial zones of plasma cell (green) accumulation. (D) Secreted APRIL (red) is associated with plasma cells (green). (E) Plasma cells (green) accumulating in the subepithelial zone express syndecan-1 (red). (F) Some secreted APRIL (red) binds (arrows) to syndecan-1 (green) expressed by plasma cells. (G) Ig isotypes (green; $5 \mu \mathrm{g} / \mathrm{ml}$ ) produced by plasma cells accumulating in APRIL-rich niches (red). Original magnification, $\times 10(\mathbf{C}) ; \times 20(\mathbf{A}$ and $\mathbf{B}) ; \times 40(\mathbf{D}, \mathbf{F}$, and $\mathbf{G}) ; \times 63(\mathbf{E})$. Figures shown are representative of 10 tonsils from patients with recurrent tonsillitis. should be noted that a majority of epithelial cells were also positive for mcl-1. Furthermore, bcl-2 was also expressed strongly by naive $B$ cells underneath subepithelial plasma cells and weakly by basal undifferentiated epithelial cells as previously reported (19, 28). Cells brightly stained by the anti-bcl- $x_{L}$ present in the epithelium did not show the same localization as plasma cells. Serial sections of the subepithelial zone showed that epithelial cells formed an ordered structure, surrounding plasma cells, thereby creating niche-like structures with a diameter that could be up to $500 \mu \mathrm{m}$, with many plasma cells inside (Figure 4). This circular build up of epithelium and plasma cells invaginated deeply into the crypt lumen, with a concomitant decrease in diameter until the structure stopped. These APRIL-rich invaginations could be close to one another so that a single crypt contained numerous niches.

We also studied MALT of the lower gut. We observed APRILproducing cells (Figure 5A) as well as secreted APRIL in the apical zone of lamina propria villi. Similarly to the tonsillar epithelium, secreted APRIL dissociated from APRIL-producing cells and was dispersed throughout the subepithelial zone (Figure 5A, inset). All APRIL-producing cells were elastase ${ }^{+} \mathrm{CD} 15^{+}$neutrophils (data not shown). Plasma cells accumulated in these APRIL-rich zones. They also expressed syndecan-1 (data not shown), allowing them to bind secreted APRIL (Figure 5B). Some secreted APRIL bound also to non-plasma cells present in this zone, most likely via an interaction with HSPG, characterized by depot-like structures. The accumulating plasma cells produced IgA and to a lesser extent IgM, but no IgG (Figure 5B). Taken together, our in situ analysis shows that plasma cells accumulate in APRIL-rich niches in MALT subepithelia from upper tonsil and lower gut in a similar manner.

Regulation of APRIL production and plasma cell accumulation in $M A L T$. We next compared APRIL production and plasma cell accumulation in tonsils with different degrees of infection. Epithelial keratinocytes from a noninfected crypt produced a basal level of APRIL that was similar to that produced by keratinocytes from chronically and acutely infected crypts (Figure 6A). We con- firmed APRIL production by epithelial cells with the detection of APRIL mRNA in cultures of primary keratinocytes derived from human skin (HEK cells) and of immortalized keratinocytes from the HACAT cell line (Figure 6B, left panel). In the former cells, quantitative RT-PCR for APRIL showed that agonists for TLR2 (peptidoglycan [PGN]), TLR4 (LPS), TLR7 (loxoribine), and TLR9 $(\mathrm{CPG})$ upregulate APRIL transcription (Figure 6B, right panel), indicating that pathogens upregulate APRIL expression in keratinocytes. The number of APRIL-producing Stalk- $1^{\text {bright }}$ neutrophils infiltrating these different epithelia varied markedly. While they were barely detectable in crypt epithelium from noninfected tonsils, their number significantly increased in chronically infected cases to reach a maximum in acutely infected specimens (Figure 6C). In infected tonsils, we also observed APRIL-producing neutrophils in blood vessels draining the organ, from where they extravasated into the extrafollicular area (Supplemental Figure 3), indicating that APRIL-producing blood neutrophils migrated toward the infected crypt epithelium after extravasation. The difference in the number of APRIL-producing neutrophils made that depots of secreted APRIL were small in control tonsils, were more pronounced during chronic infection, and were at least 10 times more prominent in acutely infected tonsils (Figure 6D). In the latter, APRIL depots covered the whole basal epithelium of the infected crypt (Supplemental Figure 4). Plasma cell accumulation varied according to the size of APRIL depots in the subepithelial zone. The number of plasma cells present in control tonsils doubled during a chronic infection and was at its maximum in acute infections (Figure 6E). In the latter case, the plasma cells completely filled the invaginations described in the previous paragraph (Supplemental Figure 4), secreting high amounts of Ig that not only covered the pathogen present in the crypt lumen but also bound to the surrounding epithelium. Hence, APRIL-rich niches are formed in the subepithelial zone of an infected crypt. These niches sustain plasma cell survival close to the pathogen, thereby enabling a lasting local humoral response. 

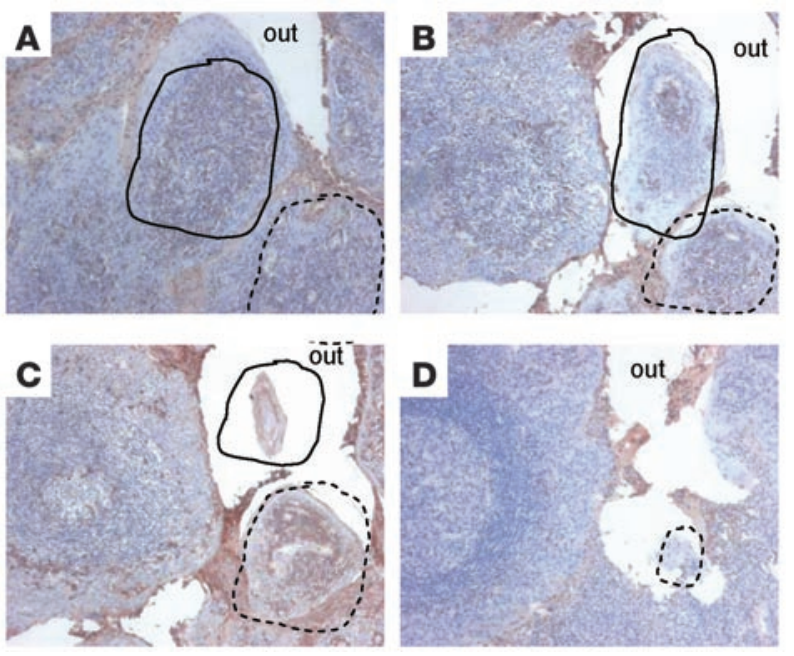

APRIL prolongs the mucosal humoral immune response. We next studied to what extent APRIL modulates humoral mucosal immunity in vivo in a mouse model. For this purpose, we measured Ig production at different times after oral vaccination with OVA in WT and APRIL-deficient C57BL/6 mice. Although at the peak of the response, 3 weeks after the last boost, the amount of OVA-specific Ig secreted by gut plasma cells was similar in WT and APRIL-deficient C57BL/6 mice (Figure 7), clear differences appeared 3 weeks later. At that moment, gut plasma cells still produced detectable antibodies in WT mice, while production had ceased in APRIL-deficient mice. This experiment shows that humoral immunity to mucosal antigens is shortened in APRIL-deficient mice, highlighting the in vivo role of APRIL for MALT plasma cell survival.

\section{Discussion}

In this report, we present evidence that APRIL-rich niches are created in mucosa to sustain local plasma cell survival. In infected mucosa, the major source of APRIL were neutrophils. This is consistent with previous reports on tumors $(22,23$, 29). Infection in the oral cavity and presence of pathogen in the tonsillar crypt resulted in a massive influx of neutrophils. We observed these neutrophils in blood vessels draining the organ, extravasated into the parenchyma, and infiltrating the tonsillar epithelium, close to the invading pathogen. Such neutrophil migration toward the tonsillar epithelium has already been documented (30) and is driven by several chemokines (CXCL-1, -5 and -8) produced by TLR-stimulated epithelial cells (31). In the absence of detectable infection, APRILproducing neutrophils were barely present in tonsils, but keratinocytes from the tonsillar epithelium produced APRIL in this condition. Pathogen-associated products stimulating TLR, known to be expressed by tonsillar epithelial cells (17, 32), upregulated this epithelial production. The basal APRIL expression by epithelial cells observed in the absence of detectable infection may reflect a constant exposure of these cells to pathogen products, since epithelial cells of tissues protected from pathogens by a physical barrier, such as the skin, do not produce APRIL unless an inflammatory reaction is ongoing (23). By contrast, epithelial cells of the small intestine did not produce APRIL. The absence of APRIL production by small intestinal epithelium warrants further investigations, since it

\section{Figure 4}

APRIL-rich niches invaginate deeply into the crypt lumen. Serial sections, at a distance of $100 \mu \mathrm{m}$ apart, of crypt epithelium starting in the tonsillar parenchyma and ending in the crypt lumen (images A-D) were stained with an anti-lg $(5 \mu \mathrm{g} / \mathrm{ml})$. Two separate invaginations (plain and dotted lines) containing cytoplasmic $\mathrm{Ig}^{+}$plasma cells are shown. Epithelium shows depots of secreted Ig. Original magnification, $\times 10$. Figures shown are representative of 5 tonsils from patients with acute tonsillitis.

also expresses TLR (33), and epithelial cells from colon further down in the gastrointestinal tract produce APRIL (16). Hence, APRIL was uniquely produced in small intestine by neutrophils that homed into villi of lamina propria, also under the influence of the chemokine CXCL-8, produced by TLR-stimulated intestinal epithelium (34). The chemoattraction of APRIL-producing neutrophils and the upregulation of APRIL production by tonsillar epithelial cells, both under the control of TLR stimulation, demonstrate that APRIL production in MALT is regulated by inflammation.

Both types of APRIL-producing cells, neutrophils and keratinocytes, secreted the molecule and the soluble product concentrated in the subepithelial zone bound to HSPG, as previously observed in tumor lesions $(22,23,29)$, retained by epithelial cells by virtue of syndecan-1 expression. These syndecan-1-expressing epithelial cells formed invaginations into the crypt lumen, wherein plasma cells accumulated. There, APRIL bound to epithelial cells could stimulate plasma cell survival in trans, as observed in our in vitro assay, with plastic-coated HSPG. APRIL also bound directly to syndecan-1 expressed by plasma cells, providing in this case a cis stimulation. These APRIL-rich niches constitute, therefore, a proper environment for plasma cell survival. Hence, mucosa epithelium has pleiotropic functions in humoral immunity. It not only induces Ig switch and retains memory B cells (35) but also promotes survival of plasma cells.
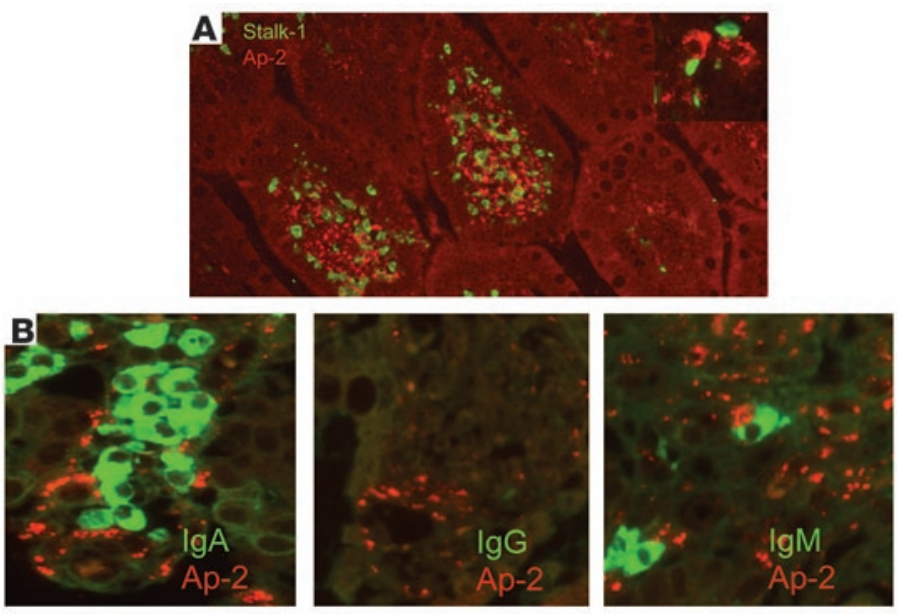

\section{Figure 5}

APRIL-rich niches are also present in human small intestine. (A) APRILproducing cells (green) and depots of secreted APRIL (red) are present in intestinal villi. Inset shows secreted APRIL (red) dissociating from APRILproducing cells (green). (B) Ig isotype $(5 \mu \mathrm{g} / \mathrm{ml}$, green) produced by plasma cells accumulating in APRIL-rich niches (red). Original magnification, $\times 20$ (A); $\times 40(\mathbf{A}$, inset, and $\mathbf{B})$. Figures shown are representative of at least 5 intestines analyzed. 
A
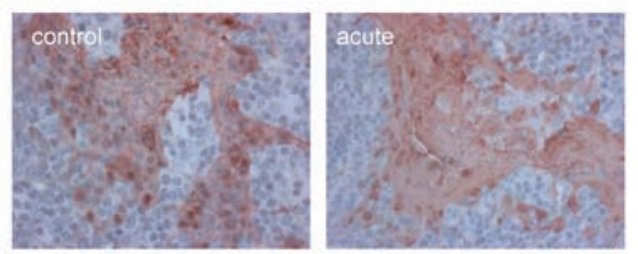

B

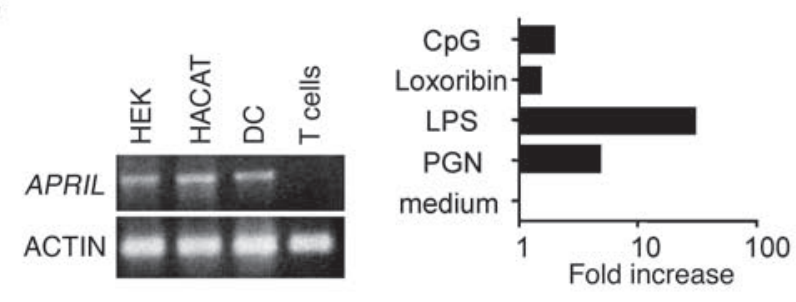

C
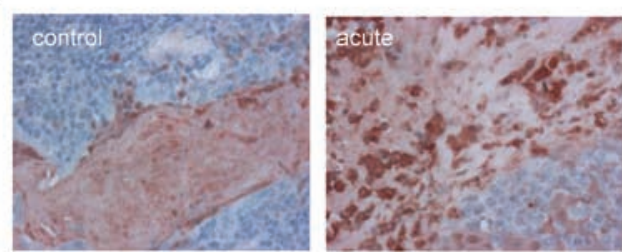

D
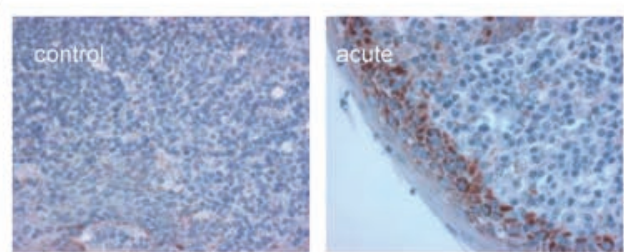

E
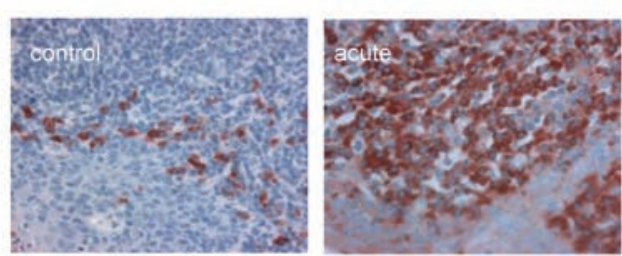

old increase

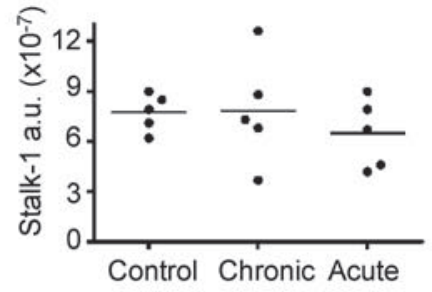

Control Chronic Acute

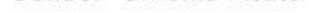

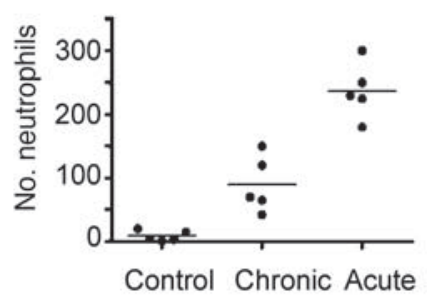
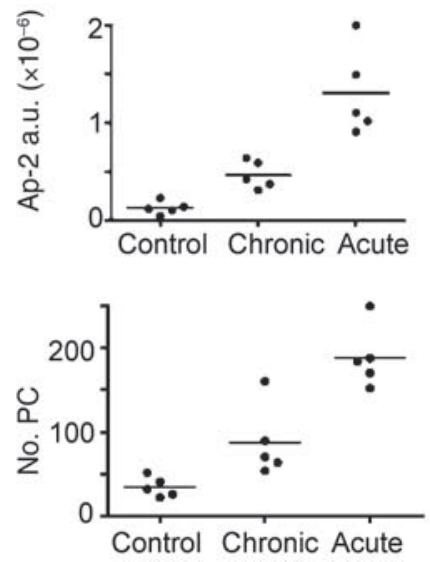

\section{Figure 6}

Inflammation regulates APRIL production in tonsils. (A) A representative example of Stalk-1 staining for crypt epithelium from control and acutely infected tonsils (left and middle panels). Quantification of the staining obtained in 5 control, 5 chronically, and 5 acutely infected tonsils is shown in the right panel. (B) APRIL transcription was analyzed by 40 cycles of RT-PCR in the indicated cells. Actin was used as housekeeping gene (left panel). Quantitative RT-PCR for APRIL mRNA was performed on epithelial HEK cells treated for 48 hours with PGN $(10 \mu \mathrm{g} / \mathrm{ml})$, LPS $(100 \mathrm{ng} / \mathrm{ml})$, Loxoribine $(250 \mu \mathrm{g} / \mathrm{ml})$, or CpG (10 $\mu \mathrm{g} / \mathrm{ml})$ (right panel). (C-E) A representative example of Stalk-1 (C), Aprily-2 (D), and anti-lg (E) stainings is shown as in A (left and middle panels). Quantification of the number of Stalk-1stained neutrophils infiltrating the crypt epithelium (C), of the Aprily-2 staining (D), and of the number of plasma cells (PCs) in the subepithelial crypt area is also shown in right panels. Original magnification, $\times 40$.
The preferential production of IgA and IgG in gut and tonsil, respectively, has been known for decades $(36,37)$. In situ specific switch factors are involved in this process $(16,17,38)$. Here we observed that plasma cells accumulate in niches underneath an epithelium that allows the full effector functions of their secreted Ig. Indeed, IgA-producing cells accumulate in APRIL-rich niches preferentially localized underneath a contiguous epithelium, so that transcytosis is required for Ig molecules to access the intestinal lumen. Notably, intestinal epithelial cells transcytose IgA but not IgG (39). In contrast, IgG-producing cells accumulated in a zone lined by a noncontiguous crypt epithelium, allowing passive diffusion of IgG molecules to reach the crypt lumen (40).

APRIL-null mice produce less IgA and IgM against T cell-independent antigens given orally as well as less IgA to T cell-dependent antigens. Although the role of APRIL in the Ig switch process (16, 41-43) explains the IgA deficiency, it does not explain the IgM defi- ciency. Here we show that APRIL also induces a survival signal, late in the $\mathrm{B}$ cell differentiation process, at the plasma cell stage. This survival signal has consequences in vivo, since humoral immunity to mucosal antigens is shortened in APRIL-deficient mice. Furthermore, the deficient production of selective Ig isotypes (IgA and IgM) in APRIL-null mice upon oral challenge is fully consistent with the accumulation of plasma cells producing IgA and IgM in APRIL-rich niches that we observed in human gut. Hence, APRIL regulates plasma cell survival in MALT, as it does in the bone marrow (44). This MALT plasma cell survival is promoted by 3 related, antiapoptotic factors, bcl-2, bcl- $\mathrm{x}_{\mathrm{L}}$, and mcl-1, each known, albeit not simultaneously, to be active in plasma cells $(9,19,44)$. This discrepancy could be due to a species (mouse versus human) or site (bone marrow versus MALT) differences. In this study, we confirmed that BCMA is the unique canonical TNF-R expressed by tonsillar plasma cells as previously reported (45). Therefore, APRIL signaling in MALT 


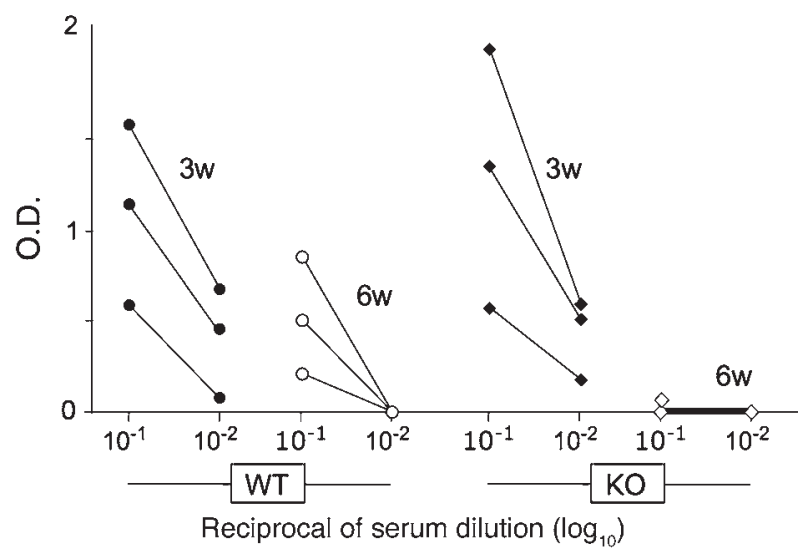

Figure 7

Mucosal humoral immunity is short lasting in APRIL-deficient mice. Groups of $A P R I L^{-1-}$ and WT mice were vaccinated orally with OVA. Secretion of OVA-specific Ig by gut plasma cells was tested 3 and 6 weeks after the last vaccination in fecal extracts by ELISA.

plasma cells is likely to be mediated by BCMA, rendering this receptor not only crucial for the longevity of bone marrow (9) but also of MALT plasma cells. BCMA expression also indicates that BAFF may also be implicated in MALT plasma cell survival, similar to the role of BAFF reported in the bone marrow $(9,46)$.

New modulatory functions in humoral immunity from the mucosal epithelium have recently been uncovered (17). We further show here that epithelial cells are also implicated in the control of mucosal plasma cell survival, either directly, by producing APRIL, or indirectly, by recruiting APRIL-producing neutrophils. By contrast, a role for neutrophils in the regulation of humoral immunity has not been established to date. The only indirect observation is that neutropenia can be linked to a decrease in serum Ig (47-49). It is known that plasma cell longevity is not defined by intrinsic characteristics but rather by environment (50). APRIL is part of this environment. In bone marrow, granulocytes produce APRIL, indicating that APRIL supply is constitutive under normal conditions (22). This may explain, at least partly, the longevity of bone marrow plasma cells (51). In contrast, the present report shows that APRIL supply in MALT is not constitutive but highly dependent on inflammation. As a result, half-life of plasma cells in MALT is more restrained as previously suggested (52).

\section{Methods}

Mouse experiments. All animal care and procedures were approved by the veterinary office of the Canton of Geneva. APRIL $L^{-/-}$mice on a C57BL/6 background were kindly provided A. Ashkenazy (Genentech Inc., San Francisco, California, USA). WT C57BL/ 6 mice were bred in house. Age- and sexmatched mice were vaccinated orally 3 times with $0.2 \mathrm{ml}$ PBS, containing $1 \mathrm{mg}$ OVA (Sigma-Aldrich) and $10 \mu \mathrm{g}$ Cholera Toxin (List Biological Laboratories Inc.) 10 days apart. Ig from fecal extracts were obtained by adding $100 \mu \mathrm{l}$ PBS with $0.2 \% \mathrm{NaN}_{3}$ to $100 \mu \mathrm{g}$ feces. After overnight vortexing and $1,500-\mathrm{g}$ centrifugation for 10 minutes at $4{ }^{\circ} \mathrm{C}$, total anti-OVA Ig in the supernatant was assessed in a standard ELISA with 96-well plates coated with OVA at $1 \mu \mathrm{g} / \mathrm{ml}$ and an anti-mouse Ig-conjugated to HRP as detection reagent.

Cells and reagents. HEK cells were isolated from human epidermis and maintained in KGM medium (Bioreba), according to Viard-Leveugle et al. (53). The following antibodies, anti-TACI (clone 1A1, rat IgG2a), antiBCMA (clone Vicky1, rat IgG2a), anti-BAFF-R (clone 11c1, IgG1), and anti-
APRIL (clone Aprily-2 and -5, both IgG1), were all from Alexis Biochemicals. The Stalk-1 antibody has been previously described (22). The anti-BAFF-R (clone 9, IgG1) and anti-TACI (A18, B1, B4, B5, C18, all IgG1) were kindly provided by Christine Ambrose (Biogen-Idec, Boston, Massachusetts, USA). ACRP-APRIL, consisting of residues 88-233 of APRIL fused to residues 16-108 of ACRP, and its control unfused ACRP were also from Alexis Biochemicals. Rabbit serum anti-syndecan-1 (H-174), anti-mcl-1 (S-19), and mouse monoclonal anti-bcl- $\mathrm{x}_{\mathrm{L}}(\mathrm{H}-5)$ were from Santa Cruz Biotechnology Inc. Anti-CD15 (clone C3D-1, IgM), anti-elastase (clone NP57, IgG1), pan-cytokeratin (rabbit IgG), and anti-CD20 (clone L26, IgG2a) antibodies were from Dako. Anti-cytokeratin 8 (clone CAM5.2, IgG2a) was from BD Biosciences. Flurochrome-conjugated anti-CD19 (clone HIB19, IgG1), anti-CD38 (clone HIT2, IgG1), and anti-bcl-2 (clone 100, IgG1) were from BD Biosciences. Alexa Fluor 488-conjugated anti-human Ig was from Molecular Probes. Isotype-specific biotinylated goat anti-IgM, IgG, and IgA were from Vector Laboratories. LPS, peptidoglycan, and CPG were from Alexis Biochemicals. Loxoribine was from Sigma-Aldrich.

B cell survival assays. Approval for research on human cells was obtained from the Ethics Committee of the Geneva University Hospital. Written informed consent was obtained for all samples according to the Declaration of Helsinki. All cultures were grown in RPMI 1640 medium, supplemented with $10 \%$ fetal calf serum and penicillin-streptomycin. FACSsorted tonsillar cells were incubated in 96-well flat-bottomed plates at a density of $2 \times 10^{5}$ cells $/ \mathrm{ml}$. Viable cells were counted by trypan-blue exclusion every day for 4 days. For trans-stimulation assay, purified HSPG (Sigma-Aldrich) was coated onto 96-well flat-bottomed plates at $1 \mu \mathrm{g} / \mathrm{ml}$, in PBS washed and incubated for 1 hour at room temperature with soluble APRIL. Flag-tagged human $\mathrm{APRIL}_{\mathrm{A} 88}$ and $\mathrm{APRIL}_{\mathrm{H} 98}$ have been previously described (22). Since APRIL ${ }_{A 88}$ could not be purified by anti-flag (M2; Sigma-Aldrich) affinity chromatography, due to inaccessibility of the flag tag, supernatants of 293-T transfected cells were used. APRIL binding was detected with Aprily-5 and peroxidase-conjugated anti-mouse serum with tetramethylbenzidine (Sigma-Aldrich) as substrate.

Flow cytometry. Cells were washed in PBS, incubated for 30 minutes at $4^{\circ} \mathrm{C}$ with primary antibodies, washed again in PBS, and incubated with secondary antibodies for an additional 30 minutes at $4{ }^{\circ} \mathrm{C}$. Cells were washed in PBS before analysis using a FACScan (BD Biosciences) and CellQuest software. For total staining, cells were fixed/permeabilized with PBS, containing $1 \%$ formaldehyde, $1 \%$ saponin as previously described (54).

Gene expression analysis. Total RNA, prepared from cells using TRIzOL Reagent (Gibco BRL), was treated with RQ1 DNAse (Promega). APRIL and actin primers for RT-PCR have been previously described (22). Quantitative RT-PCR was done as previously described with a light cycler System (Roche Diagnostics) and the Quantitect SYBR Green PCR kit solution (QIAGEN) (55). For hAPRIL, 5'-ATGGGTCAGGTGGTGTCTCG-3' and 5'-ATGGGTCAGGTGGTGTCTCG-3' were used as forward and reverse primers, respectively. The control GAPDH primers were 5'-TCATGGGTGTGAACCATGAGA-3' (forward) and 5'-GCTAAGCAGTTGGTGGTGCA-3' (reverse). APRIL cDNA was used to establish the APRIL standard curve. Total reverse transcribed RNA from Hela cells was used for the GAPDH standard curve. Results were normalized to GAPDH and expressed as arbitrary units. Amplification was done in duplicates and experiments were performed twice.

Immunobistochemistry. Tonsils were obtained from patients with recurrent tonsillitis associated-infections, from patients during an acute infection, and from patients with snoring problems. For the latter, the patients had not experienced any infections of the tonsils for the previous 2 years and had not been treated with antibiotics during the last 3 months preceding surgery. There was no clinical sign of infection at the time of tonsillectomy for these latter patients. Small intestine specimens were obtained from patients bearing Merckel cell carcinoma outside tumor lesions. Specimens 
with signs of an ongoing humoral response (presence of Peyer's patches) were selected. After deparaffinization, sections were incubated for $10 \mathrm{~min}$ utes in methanol plus $0.6 \%$ hydrogen peroxide at room temperature, followed by washing with phosphate-buffered saline solution. Antigen retrieval was performed by boiling slides once in $0.01 \mathrm{M}$ citrate buffer, $\mathrm{pH}$ 6.0, for 3 minutes. Incubation with antibodies was carried out for 1 hour at room temperature. For immunoperoxidase staining, secondary reagents were goat anti-rabbit or anti-mouse IgG sera conjugated to biotin (Jackson ImmunoResearch Laboratories Inc.), followed by streptABComplex/HRP (Dako) and 3-amino-9-ethylcarbazole substrate (Sigma-Aldrich). For antibcl-2, bcl- $x_{\mathrm{L}}$, and mcl-1, the EnVision plus HRP (Dako) was used for revelation with 3 periods of boiling in EDTA buffer, $\mathrm{pH}$ 8.6, for antigen retrieval. For 2-color immunofluorescence stainings, fluorochrome-conjugated isotype-specific goat anti-mouse sera (Jackson ImmunoResearch Laboratories Inc.) and goat Ig anti-rabbit IgG (Molecular Probes) conjugated to phycoerythrin were used. Images were visualized under light or fluorescent microscopy with Axiophot 1 (Carl Zeiss AG), captured with an Axiocam (Carl Zeiss AG) color CCD camera, and treated on a Pentium III computer with AxioVision software (Carl Zeiss AG). In some experiments, confocal analyses with an LSM 510 (Carl Zeiss AG) were performed. For numeration of neutrophils and plasma cells, image acquisition was done with the $\times 40$ objective, and stained cells were counted in the entire field, corresponding to a tissue area of $30 \mathrm{~mm}^{2}$. For the Stalk-1 and Aprily-2 signal quantification, images acquired with the $\times 40$ objective were processed in MetaMorph Image Analysis software (Molecular Devices). Color thresholds were selected in the Hue, Saturation, Intensity (HSI) space. Saturation values obtained in the thresholded field were added, logged to a spreadsheet, and expressed in arbitrary units.

\section{Acknowledgments}

We thank S. Izui for critical reading of the manuscript. The expertise of Sergei Startchik in signal quantification is greatly acknowledged. This work was supported by the Dr Henri Dubois-Ferrière Dinu Lipatti Foundation and the Swiss National Science Foundation.

Received for publication August 28, 2007, and accepted in revised form May 28, 2008.

Address correspondence to: Bertrand Huard, Department of PathoImmunology, University Medical Center, 1 rue Michel Servet, $\mathrm{CH}$ 1211 Geneva 4, Switzerland. Phone: (41) 22-379-58-11; Fax: (41) 22-379-58-02; E-mail: bertrand.huard@medecine.unige.ch.

Stéphane Durual's present address is: Department of Prosthodontics/Biomaterials, School of Dental Medicine, University of Geneva, Geneva, Switzerland.
1. Barreto, V.M., Ramiro, A.R., and Nussenzweig, M.C. 2005. Activation-induced deaminase: controversies and open questions. Trends Immunol. 26:90-96.

2. Schwickert, T.A., et al. 2007. In vivo imaging of germinal centres reveals a dynamic open structure. Nature. 446:83-87.

3. Manz, R.A., Hauser, A.E., Hiepe, F., and Radbruch, A. 2005. Maintenance of serum antibody levels. Annu. Rev. Immunol. 23:367-386.

4. Cassese, G., et al. 2003. Plasma cell survival is mediated by synergistic effects of cytokines and adhesion-dependent signals. J. Immunol. 171:1684-1690.

5. Kunkel, E.J., and Butcher, E.C. 2003. Plasma-cell homing. Nat. Rev. Immunol. 3:822-829.

6. Pabst, O., et al. 2004. Chemokine receptor CCR9 contributes to the localization of plasma cells to the small intestine. J. Exp. Med. 199:411-416.

7. Kalled, S.L., Ambrose, C., and Hsu, Y.M. 2005. The biochemistry and biology of BAFF, APRIL and their receptors. Curr. Dir. Autoimmun. 8:206-242.

8. Ingold, K., et al. 2005. Identification of proteoglycans as the APRIL-specific binding partners. J. Exp. Med. 201:1375-1383.

9. O'Connor, B.P., et al. 2004. BCMA is essential for the survival of long-lived bone marrow plasma cells. J. Exp. Med. 199:91-98.

10. Day, E.S., et al. 2005. Selectivity of BAFF/BLyS and APRIL for binding to the TNF family receptors BAFFR/BR3 and BCMA. Biochemistry. 44:1919-1931

11. Thompson, J.S., et al. 2001. BAFF-R, a newly identified TNF receptor that specifically interacts with BAFF. Science. 293:2108-2111.

12. Hendriks, J., et al. 2005. Heparan sulfate proteoglycan binding promotes APRIL-induced tumor cell proliferation. Cell Death Differ. 12:637-648.

13. Schiemann, B., et al. 2001. An essential role for BAFF in the normal development of B cells through a BCMA-independent pathway. Science. 293:2111-2114.

14. Varfolomeev, E., et al. 2004. APRIL-deficient mice have normal immune system development. Mol. Cell. Biol. 24:997-1006.

15. Castigli, E., et al. 2004. Impaired IgA class switching in APRIL-deficient mice. Proc. Natl. Acad. Sci. U. S. A. 101:3903-3908.
16. He, B., et al. 2007. Intestinal bacteria trigger T cellindependent immunoglobulin A(2) class switching by inducing epithelial-cell secretion of the cytokine APRIL. Immunity. 26:812-826.

17. Xu, W., et al. 2007. Epithelial cells trigger frontline immunoglobulin class switching through a pathway regulated by the inhibitor SLPI. Nat. Immunol. 8:294-303.

18. Salzer, U., et al. 2005. Mutations in TNFRSF13B encoding TACI are associated with common variable immunodeficiency in humans. Nat. Genet. 37:820-828.

19. Merville, P., et al. 1996. Bcl-2+ tonsillar plasma cells are rescued from apoptosis by bone marrow fibroblasts. J. Exp. Med. 183:227-236.

20. Medina, F., Segundo, C., Campos-Caro, A., Gonzalez-Garcia, I., and Brieva, J.A. 2002. The heterogeneity shown by human plasma cells from tonsil, blood, and bone marrow reveals graded stages of increasing maturity, but local profiles of adhesion molecule expression. Blood. 99:2154-2161.

21. Withers, D.R., et al. 2007. T cell-dependent survival of CD20+ and CD20- plasma cells in human secondary lymphoid tissue. Blood. 109:4856-4864.

22. Schwaller, J., et al. 2007. Neutrophil-derived APRIL concentrated in tumor lesions by proteoglycans correlates with human B-cell lymphoma aggressiveness. Blood. 109:331-338.

23. Mhawech-Fauceglia, P., et al. 2006. The source of APRIL up-regulation in human solid tumor lesions. J. Leukoc. Biol. 80:697-704.

24. Clark, M.A., Wilson, C., Sama, A., Wilson, J.A., and Hirst, B.H. 2000. Differential cytokeratin and glycoconjugate expression by the surface and crypt epithelia of human palatine tonsils. Histochem. Cell Biol. 114:311-321.

25. Perry, M., and Whyte, A. 1998. Immunology of the tonsils. Immunol. Today. 19:414-421.

26. Brandtzaeg, P., and Johansen, F.E. 2005. Mucosal B cells: phenotypic characteristics, transcriptional regulation, and homing properties. Immunol. Rev. 206:32-63.

27. Tedder, T.F., and Engel, P. 1994. CD20: a regulator of cell-cycle progression of B lymphocytes. Immunol. Today. 15:450-454.

28. Krajewski, S., et al. 1995. Immunohistochemical analysis of Mcl-1 protein in human tissues. Differ- ential regulation of $\mathrm{Mcl}-1$ and $\mathrm{Bcl}-2$ protein production suggests a unique role for Mcl- 1 in control of programmed cell death in vivo. Am. J. Pathol. 146:1309-1319.

29. Schwaller, J., et al. 2007. Paracrine promotion of tumor development by the TNF ligand APRIL in Hodgkin's disease. Lenkemia. 21:1324-1327.

30. Ebenfelt, A., and Ivarsson, M. 2001. Neutrophil migration in tonsils. J. Anat. 198:497-500.

31. Sachse, F., Ahlers, F., Stoll, W., and Rudack, C. 2005. Neutrophil chemokines in epithelial inflammatory processes of human tonsils. Clin. Exp. Immunol. 140:293-300.

32. Claeys, S., et al. 2003. Human beta-defensins and toll-like receptors in the upper airway. Allergy. 58:748-753.

33. Chabot, S., Wagner, J.S., Farrant, S., and Neutra, M.R. 2006. TLRs regulate the gatekeeping functions of the intestinal follicle-associated epithelium. J. Immunol. 176:4275-4283.

34. Kucharzik, T., and Williams, I.R. 2002. Neutrophil migration across the intestinal epithelial barrier-summary of in vitro data and description of a new transgenic mouse model with doxycycline-inducible interleukin-8 expression in intestinal epithelial cells. Pathobiology. 70:143-149.

35. Liu, Y.J., et al. 1995. Memory B cells from human tonsils colonize mucosal epithelium and directly present antigen to $T$ cells by rapid up-regulation of B7-1 and B7-2. Immunity. 2:239-248.

36. Conley, M.E., and Delacroix, D.L. 1987. Intravascular and mucosal immunoglobulin A: two separate but related systems of immune defense? Ann. Intern. Med. 106:892-899.

37. Nadal, D., Soh, N., Schlapfer, E., Bernstein, J.M., and Ogra, P.L. 1992. Distribution characteristics of immunoglobulin-secreting cells in adenoids. Relationship to age and disease. Int. J. Pediatr. Otorhinolaryngol. 24:121-130.

38. Fagarasan, S., Kinoshita, K., Muramatsu, M., Ikuta, K., and Honjo, T. 2001. In situ class switching and differentiation to IgA-producing cells in the gut lamina propria. Nature. 413:639-643.

39. Brandtzaeg, P., and Prydz,H. 1984. Direct evidence for an integrated function of J chain and secretory component in epithelial transport of immunoglobulins. Nature. 311:71-73. 
40. Ogra, P.L., Faden, H., and Welliver, R.C. 2001. Vaccination strategies for mucosal immune responses. Clin. Microbiol. Rev. 14:430-445.

41. Litinskiy, M.B., et al. 2002. DCs induce CD40-independent immunoglobulin class switching through BLyS and APRIL. Nat. Immunol. 3:822-829.

42. Castigli, E., et al. 2005. TACI and BAFF-R mediate isotype switching in B cells. J. Exp. Med. 201:35-39.

43. Sakurai, D., et al. 2007. TACI regulates IgA production by APRIL in collaboration with HSPG. Blood. 109:2961-2967.

44. Belnoue, E., et al. 2008. APRIL is critical for plasmablast survival in the bone marrow and poorly expressed by early life bone marrow stromal cells. Blood. 111:2755-2764.

45. Ng, L.G., et al. 2004. B cell-activating factor belonging to the TNF family (BAFF)- $R$ is the principal BAFF receptor facilitating BAFF costimulation of circulating $\mathrm{T}$ and B cells. J. Immunol. 173:807-817.
46. Benson, M.J., et al. 2008. Cutting edge: the dependence of plasma cells and independence of memory B cells on BAFF and APRIL. J. Immunol. 180:3655-3659.

47. Sugita, K., et al. 1993. An infant with both autoimmune neutropenia and idiopathic thrombocytopenia with IgG2/IgA deficiency. Int. J. Hematol. 57:27-30.

48. Karlstrom, R., Gustafson, R., and Palmblad, J. 2001. Chronic mild neutropenia in adults: relation to IgG3 deficiency and infection susceptibility. J. Intern. Med. 250:342-347.

49. Cham, B., Bonilla, M.A., and Winkelstein, J. 2002. Neutropenia associated with primary immunodeficiency syndromes. Semin. Hematol. 39:107-112.

50. Sze, D.M., Toellner, K.M., Garcia de Vinuesa, C., Taylor, D.R., and MacLennan, I.C. 2000. Intrinsic constraint on plasmablast growth and extrinsic limits of plasma cell survival. J. Exp. Med. 192:813-821.
51. Manz, R.A., Thiel, A., and Radbruch, A. 1997. Lifetime of plasma cells in the bone marrow. Nature. 388:133-134.

52. Moser, K., Tokoyoda, K., Radbruch, A., MacLennan, I., and Manz, R.A. 2006. Stromal niches, plasma cell differentiation and survival. Curr. Opin. Immunol. 18: $265-270$.

53. Viard-Leveugle, I., et al. 2003. Intracellular localization of keratinocyte Fas ligand explains lack of cytolytic activity under physiological conditions. J. Biol. Chem. 278:16183-16188.

54. Huard, B., et al. 2004. BAFF production by antigenpresenting cells provides $\mathrm{T}$ cell co-stimulation. Int. Immunol. 16:467-475.

55. Arlettaz, L., Degermann, S., De Rham, C., Roosnek, E., and Huard, B. 2004. Expression of inhibitory KIR is confined to CD8+ effector T cells and limits their proliferative capacity. Eur. J. Immunol. 34:3413-3422. 